\title{
Distinct signatures of host-microbial meta-metabolome and gut microbiome in two C57BL/6 strains under high-fat diet
}

\author{
Alesia Walker ${ }^{1}$, Barbara Pfitzner ${ }^{2}$, Susanne Neschen ${ }^{3}$, Melanie Kahle ${ }^{3}$, Mourad Harir ${ }^{1}$, \\ Marianna Lucio ${ }^{1}$, Franco Moritz ${ }^{1}$, Dimitrios Tziotis ${ }^{1}$, Michael Witting ${ }^{1}$, \\ Michael Rothballer ${ }^{2}$, Marion Engel ${ }^{4}$, Michael Schmid ${ }^{2}$, David Endesfelder ${ }^{5}$, \\ Martin Klingenspor ${ }^{6}$, Thomas Rattei ${ }^{7}$, Wolfgang zu Castell ${ }^{5}$, Martin Hrabé de Angelis ${ }^{3}$, \\ Anton Hartmann ${ }^{2}$ and Philippe Schmitt-Kopplin ${ }^{1,8}$ \\ ${ }^{1}$ Research Unit Analytical BioGeoChemistry, Helmholtz Zentrum München, German Research Center for \\ Environmental Health, Neuherberg, Germany; ${ }^{2}$ Research Unit Microbe-Plant Interactions, Research Group \\ Molecular Microbial Ecology, Helmholtz Zentrum München, German Research Center for Environmental \\ Health, Neuherberg, Germany; ${ }^{3}$ Institute of Experimental Genetics, Helmholtz Zentrum München, German \\ Research Center for Environmental Health, Neuherberg, Germany; ${ }^{4}$ Research Unit Environmental Genomics, \\ Helmholtz Zentrum München, German Research Center for Environmental Health, Neuherberg, Germany; \\ ${ }^{5}$ Scientific Computing Research Unit, Helmholtz Zentrum München, German Research Center for \\ Environmental Health, Neuherberg, Germany; ${ }^{6}$ Technische Universität München, Molecular Nutritional \\ Medicine, Else Kröner-Fresenius Center and ZIEL Research Center for Nutrition and Food Sciences, \\ Freising-Weihenstephan, Germany; ${ }^{7}$ Department of Computational Systems Biology, University of \\ Vienna, Vienna, Austria and ${ }^{8}$ Technische Universität München, Chair of Analytical Food Chemistry, \\ Freising-Weihenstephan, Germany
}

A combinatory approach using metabolomics and gut microbiome analysis techniques was performed to unravel the nature and specificity of metabolic profiles related to gut ecology in obesity. This study focused on gut and liver metabolomics of two different mouse strains, the C57BL/6J (C57J) and the C57BL/6N (C57N) fed with high-fat diet (HFD) for 3 weeks, causing dietinduced obesity in C57N, but not in C57J mice. Furthermore, a 16S-ribosomal RNA comparative sequence analysis using 454 pyrosequencing detected significant differences between the microbiome of the two strains on phylum level for Firmicutes, Deferribacteres and Proteobacteria that propose an essential role of the microbiome in obesity susceptibility. Gut microbial and liver metabolomics were followed by a combinatory approach using Fourier transform ion cyclotron resonance mass spectrometry (FT-ICR-MS) and ultra performance liquid chromatography time of tlight MS/MS with subsequent multivariate statistical analysis, revealing distinctive host and microbial metabolome patterns between the $\mathrm{C} 57 \mathrm{~J}$ and the $\mathrm{C} 57 \mathrm{~N}$ strain. Many taurine-conjugated bile acids (TBAs) were significantly elevated in the cecum and decreased in liver samples from the C57J phenotype likely displaying different energy utilization behavior by the bacterial community and the host. Furthermore, several metabolite groups could specifically be associated with the C57N phenotype involving fatty acids, eicosanoids and urobilinoids. The mass differences based metabolite network approach enabled to extend the range of known metabolites to important bile acids (BAs) and novel taurine conjugates specific for both strains. In summary, our study showed clear alterations of the metabolome in the gastrointestinal tract and liver within a HFD-induced obesity mouse model in relation to the host-microbial nutritional adaptation.

The ISME Journal (2014) 8, 2380-2396; doi:10.1038/ismej.2014.79; published online 6 June 2014

Subject Category: Integrated genomics and post-genomics approaches in microbial ecology

Keywords: obesity; metabolomics; bile acids; mass spectrometry; gut microbiome; 16S-rRNA amplicon pyrosequencing

Correspondence: P Schmitt-Kopplin, Research Unit Analytical BioGeoChemistry (BGC), Helmholtz Zentrum München, German Research Center for Environmental Health, Ingolstädter Landstrasse 1, 85764 Neuherberg, Germany.

E-mail: schmitt-kopplin@helmholtz-muenchen.de

Received 3 December 2013; revised 25 March 2014; accepted 7 April 2014; published online 6 June 2014

\section{Introduction}

The gut microbiome is a complex microbial ecosystem containing bacteria, archaea, fungi, viruses and eukaryotes that are functionally involved in various biological processes, thus, affecting directly and indirectly host physiology (Neish, 2009; David 
et al., 2013). Several studies demonstrated that the gut microbiome composition changes depend on diet, host genotype as well as intestinal and metabolic diseases (Spor et al., 2011). Weight gain and obesity are risk factors for development of insulin resistance and type 2 diabetes and were linked to changes in the gut community composition in different independent studies (Kahn and Flier, 2000; Turnbaugh et al., 2006; Bäckhed et al., 2007; Caballero, 2007). Studies were undertaken using germ free, conventional and colonized mice in order to understand the role of gut microbiome and its contribution to weight gain or obesity (Bäckhed et al., 2004). For instance, colonization of germ-free wild-type mice with normal body fat mass with microbiota from genetically $(o b / o b)$ or diet-induced obese donor mice accelerated body fat accumulation in the recipients (Turnbaugh et al., 2006; Bäckhed et al., 2007). These observations suggest a strong impact of the gut microbial community on energy balance regulation. A widely used method to analyse gut microbiome structures is 454 pyrosequencing of 16S-ribosomal RNA (rRNA) genes to explore bacterial community compositions involved in pathophysiology of metabolic disorders. In addition to the knowledge about the involved microbiome in such a scenario of a metabolic disease, the consequential question about the functional impact of these communities can be answered by non-targeted metabolomics, which is a part of systems biology. This is a hypothesis-free and unbiased approach that enables to detect changes in metabolite patterns during pathophysiological states, drug treatments or nutritional changes (Nicholson et al., 1999). Electrospray ionization Fourier transform ion cyclotron resonance mass spectrometry in positive and negative mode $((+/-)$ ESI FT-ICR-MS) allows to profile and describe globally the metabolome changes associated with metabolic conditions based on measurements of several thousands of mass signals simultaneously due to high sensitivity, resolution and mass accuracy (Jansson et al., 2009). Most of the metabolomics studies relevant in the research of obesity were performed with body fluids that cover only a small subset of metabolites (Xie et al., 2012). Only few metabolomics studies applied MS based techniques to investigate the metabolite patterns in intestinal/fecal and liver samples following exposure to different diets and disease conditions to discover the role and influence on the metabolome as a readout (Jansson et al., 2009; Antunes et al., 2011; Baur et al., 2011; Swann et al., 2011; Matsumoto et al., 2012). This study is performed by using two C57BL/6 mouse strains (C57BL/6J (C57J) and C57BL/6N (C57N)) exposed to a high-fat diet (HFD) for 3 weeks. Apart from several singlenucleotide polymorphisms, the two strains differ in the nicotinamide nucleotide transhydrogenase gene, which bears a missense mutation, detected in the strain C57J. Moreover, the strain phenotype, such as body weight, insulin resistance, blood glucose levels were strongly dependent on the applied diet (Toye et al., 2005; Mekada et al., 2009; Nicholson et al., 2010; Montgomery et al., 2013).

The aim was to reveal the impact in the host microbial metabolome and microbiome patterns related to body weight gain after 3 weeks in the C57N strain applying 454 pyrosequencing and a non-targeted metabolomics approach to assess the role of gut ecology and host in obesity.

\section{Materials and methods}

Diet intervention, body composition analysis and sample collection

C57N (Taconic, Ry, Denmark) and C57J were bred and housed under standard vivarium conditions (12:12 light-dark-cycle). At an age of 14 weeks, male mice were single housed (cages included domehouse) on grid panels in the same room. Males of each strain were litter-matched, body weight was recorded and mice allocated into two groups. The strains were switched to a HFD (Ssniff, Soest, Germany; $24.3 \mathrm{~kJ} \mathrm{~g}^{-1}$ ) rich in safflower-oil for 3 weeks. Food was exchanged every 2nd to 3rd day. Body mass was measured 1 day before the start and at the end of the experiment in each mouse. After 3 weeks, mice were killed with an isoflurane overdose. The gastrointestinal tract of each animal was removed and the luminal content of the cecum of each mouse was collected and was equally divided for microbiome and meta-metabolome analyses, immediately snap-frozen in liquid nitrogen and preserved in $-80^{\circ} \mathrm{C}$ before further experiments. In addition, liver samples were collected for metabolomics analyses. All animals received humane care according to criteria outlined in the NAS 'Guide for the Care and Use of Laboratory Animals'. Animal experiments were approved by the Upper-Bavarian district government (Regierung von Oberbayern, Gz.55.2-1-54-2532-70-07, Gz. 55.2-1-54-2532-4-11).

\section{S-rRNA gene pyrosequencing}

Approximately $30 \mathrm{mg}$ of cecal luminal contents of C57J $(n=8)$ and C57N $(n=11)$ mice were used for microbiome analysis. Total bacterial genomic DNA was extracted using NucleoSpin for Soil Kit (Macherey-Nagel, Dueren, Germany) following the manufacturer's instructions. Amplification of the V6-V9 region was performed according to Timmers et al. (2012). Briefly, for PCR 16S-rRNA gene forward primer 926F (5'-AAACTYAAAKGAATTGACGG-3') (Lane, 1991) attached to the Roche A adapter for 454-library construction and reverse primer 630R (5'-CAKAAAGGAGGTGATCC-3') (Juretschko et al., 1998) attached to the Roche $B$ adapter were used. For multiplexing purposes, each primer included a 10-nt barcode sequence. Three independent PCRs were performed for each sample with Fast Start High Fidelity PCR System (Roche, Mannheim, Germany) containing $20 \mathrm{ng}$ of DNA with an optimal annealing 
temperature of $50{ }^{\circ} \mathrm{C}$ and 22 cycles. PCR reactions were pooled and purified using a QiaQuick PCR Purification Kit (Qiagen, Hilden, Germany). After quantification using a Quant-iT PicoGreen dsDNA quantification kit (Invitrogen, Paisley, UK), samples were equally pooled. The sequencing of the partial 16S-rRNA genes was performed on a 454 GS FLX Titanium system (Roche). Amplicons were sequenced as recommended in the instructions of the manufacturer for amplicon sequencing. Sequences were processed and data were analysed according to the 454 Schloss standard operating procedure (http:// www.mothur.org/wiki/Schloss_SOP) (Schloss et al., 2011) with the software mothur v.1.29.0 (Schloss et al., 2009). Total bacterial diversity was estimated analysing reverse sequences only. Reads were denoised, quality filtered and trimmed. Sequences with similarity $>97 \%$ were combined to one operational taxonomic unit (OTU). For taxonomy analysis, sequences were aligned against Silva SEED alignment database (Quast et al., 2013), chimeras were removed using uchime implementation (Edgar et al., 2011) in mothur and taxonomic assignment was done using RDP trainset 7 with a cut off of $80 \%$ (Cole et al., 2009). For equal comparison, subsamples of 6486 sequences of each sample were created. Within the significantly different families of the four abundant phyla 1-2 OTUs, showing highest abundances, were aligned against Silva database (http://www.arb-silva.de) using SILVA Incremental Aligner (SINA) (v.1.2.11) (Pruesse et al., 2012) and then imported to ARB (SILVA SSU database, release 111) (Ludwig et al., 2004). The closest cultivated relatives were selected and included for calculation of similarity distance matrices.

Multivariate and statistical analysis of microbiome data was performed on $\mathrm{R}$ platform ( $\mathrm{R}$ version 2.15.1; http://www.r-project.org) using the packages vegan (Dixon, 2003) and ade4 (Dray and Dufour, 2007) and custom R scripts. All OTUs with $<0.01 \%$ of the total abundance were excluded from the analysis. The measured abundances were Hellinger transformed (Ramette, 2007). Differences between groups (on phylum, family and OTU level) were analysed using Wilcoxon-Mann-Whitney test with Benjamini-Hochberg correction for multiple testing (Benjamini and Hochberg, 1995). Differences with an adjusted $P \leqslant 0.05$ were considered to be statistically significant. The means of sequence abundances of the two strains were expressed as percentages and displayed in stacked barplots. For the statistical analysis on OTU level, non-parametric multivariate analysis of variance analysis (npMANOVA) was performed based on Bray-Curtis distance measure.

\section{Non-targeted metabolomics}

Metabolite extraction of cecal and liver samples. Cecal samples $(\sim 10 \mathrm{mg})$ of C57J $(n=10)$ and C57N $(n=12)$ were placed in ceramic bead tubes (NucleoSpin Bead Tubes, Macherey-Nagel, Dueren, Germany) combined with a metal bead (Qiagen, Hilden, Germany) on dry ice. After the addition of $1 \mathrm{ml}$ of cold $\left(-20^{\circ} \mathrm{C}\right)$ methanol (LC-MS Chromasolv, Fluka, Sigma-Aldrich, St Louis, MO, USA), the homogenization was performed in TissueLyser II (Qiagen) for $5 \mathrm{~min}$ at a rate of $30 \mathrm{~Hz}$ to homogenize and disrupt the bacterial cells and extract the metabolites of the gut microbiome. Then, the samples were centrifuged two times at 14000 r.p.m. for $10 \mathrm{~min}$ at $4{ }^{\circ} \mathrm{C}$ and the supernatant was collected for MS analysis. About $50 \mathrm{mg}$ of liver were grounded and homogenized under liquid nitrogen. Metabolite extraction was performed by applying same metabolite extraction procedure, done with cecal samples.

FT-ICR-MS analysis. Non-targeted metabolomics was performed by means of a FT-ICR-MS (Bruker Daltonik GmbH, Bremen, Germany), equipped with a 12 Tesla superconducting magnet and an Apollo II ESI source. Before the measurements, the external calibration of the FT-ICR-MS was performed with clusters of arginine by using $5 \mathrm{mgl}^{-1}$ arginine solution in methanol for negative ionization mode resulting in calibration errors below 0.1 p.p.m. for four selected $\mathrm{m} / \mathrm{z}$ signals of arginine clusters $(\mathrm{m} / \mathrm{z}$ 173.10440, 347.21607, 521.32775 and 695.43943). The infusion of the extracted samples into the mass spectrometer was performed in a randomized order with a flow rate of $120 \mu \mathrm{h} \mathrm{h}^{-1}$ by using a Gilson autosampler system (Gilson, Inc., Middleton, WI, USA). The spectra were acquired with a time domain of 2 megawords in a mass range of $140-1000 \mathrm{~m} / \mathrm{z}$ with a resolving power of 400000 at $\mathrm{m} / \mathrm{z} 400$. The ion accumulation time was set to $0.5 \mathrm{~s}$ and time of flight was set to $0.8 \mathrm{~ms}$. Spectra were accumulated for 450 scans in both modes for all samples. The mass spectrometer was set to a capillary voltage and a spray shield voltage to $(+/-) 4000 \mathrm{~V}$ and $(+/-) 500 \mathrm{~V}$, respectively. Drying gas flow rate and temperature was set to $4 \mathrm{lmin}^{-1}$ and $200{ }^{\circ} \mathrm{C}$ and nebulizer gas flow rate was set to 1.0 bar. The raw spectra were processed with Data Analysis Version 4.0 SP2 (Bruker Daltonik GmbH, Bremen, Germany). Different pre-processing steps have been undertaken before the statistical analysis. First, the raw spectra were internally calibrated with reference lists with given masses for both modes with an error below 0.1 p.p.m. The calibrated mass spectra were exported into ASCI files with a signalto-noise ratio of 4 and a relative intensity threshold of $0.01 \%$ covering all signals above background noise. The acquired peak lists were aligned through an in-house program (Lucio et al., 2010). The alignment was performed by clustering the masses within an error window of 1 p.p.m and concatenating consequently their intensities. Identification experiments were performed with ultra performance liquid chromatography time of flight MS/MS (detailed information in Supplementary 
Information). Data processing, statistical analysis and data annotation of metabolome data are described in Supplementary Information.

\section{Results}

General

The C57N strain showed higher body weight changes compared with the C57J strain because of 3 weeks of HFD feeding, shown in Figure 1a. We hypothesized that the observed body weight changes occurred between the strains are associated with different patterns of gut microbiome structure and metabolite patterns in cecum and liver organ. In mammalian system, a cecal metabolome is shaped by both bacterial and host compounds, thus termed as meta-metabolome, while the liver metabolome reflects majorly the host metabolism. Both organs were used to reflect the metabolism between C57J and $\mathrm{C} 57 \mathrm{~N}$ mice on HFD.

\section{Description of the differences in the gut microbiome} structure

Microbiome studies were based on 454 pyrosequencing of 16S-rRNA genes. To compare community
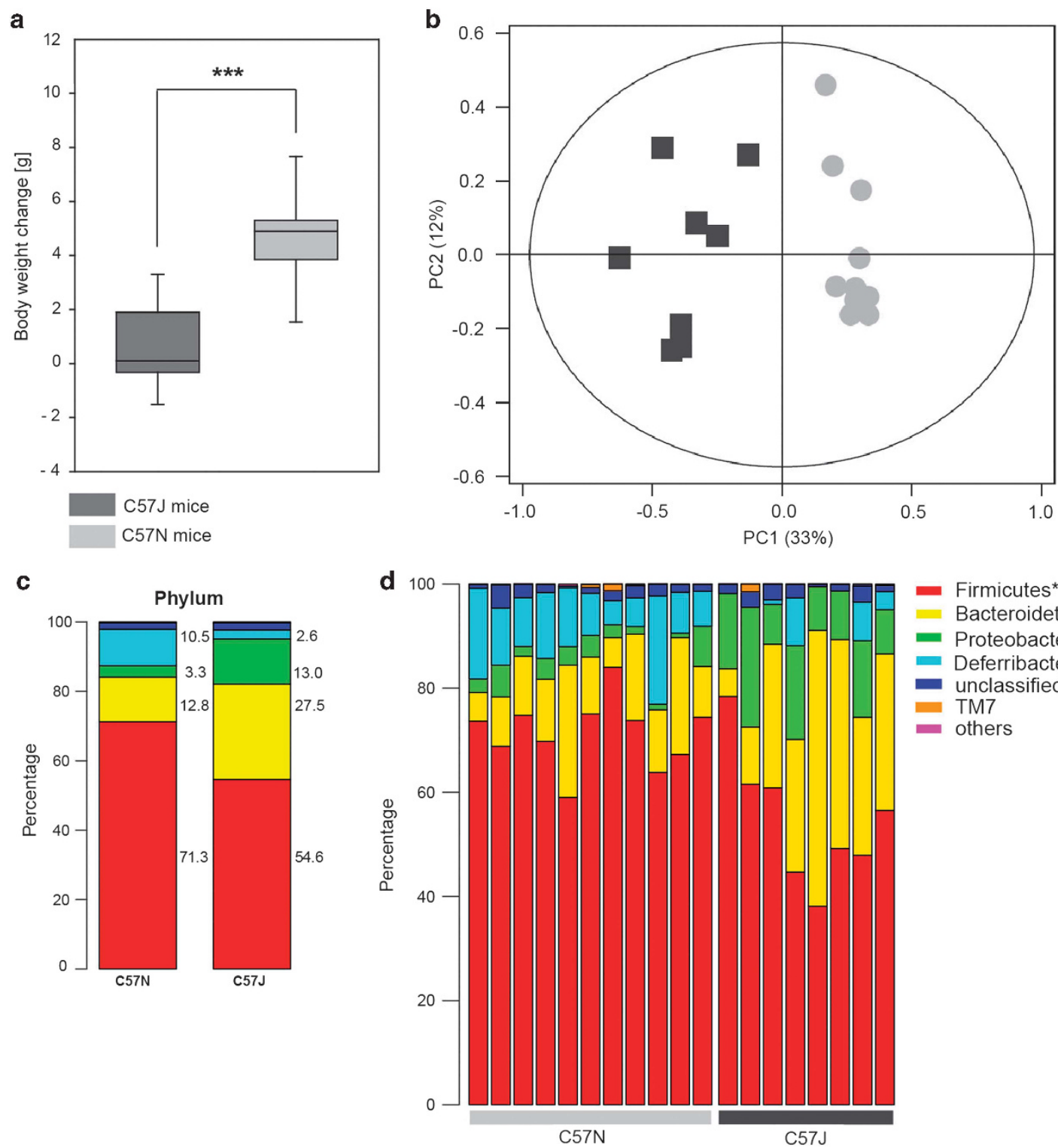

Figure 1 HF feeding of 3 weeks impacts body weight in C57N mice compared with the C57J and influences the bacterial community in the gut. (a) Significant body weight changes between C57J and C57N mice, $P$-value ${ }^{*}<0.05,{ }^{*}<0.01,{ }^{*} *<0.001$, (Student's $t$-test) (b) Bacterial community changes between C57J (black rectangle) and C57N (gray dots)) mice, originated from principal component analysis (PCA). The analysis was performed on data based on relative abundance and Hellinger transformed 16S-rRNA data of classified OTUs with >97\% identity. (c) Cecal bacterial profiles on phylum level of C57J and C57N mice, displaying relative abundance of partial sequences of bacterial 16S-rRNA genes. Individual phylum levels of each mouse are shown in d. Classification was done at the phylum level using mothur with a modified 16S-rRNA gene database from RDP: P-value: ${ }^{*}<0.05,{ }^{* *}<0.01,{ }^{* *}<0.001$ (Wilcoxon-Mann-Whitney test). 
patterns, a principal component analysis was used. A scatter plot based on principal component analysis scores obtained from the sequences at OTU level with $>97 \%$ similarity showed a clear separation of the community composition between the groups (Figure 1b). In addition, a npMANOVA, based on Bray-Curtis distance also confirmed significant differences $(P$-value $<0.001)$ between bacterial community composition on OTU level. Around $99 \%$ of the total bacterial abundance was classified into eight phyla, while the rest was allocated to various unclassified bacteria. As shown in Figure 1c, Firmicutes was the most abundant phylum in all samples, accounting for 55\% (in C57J mice) and $71 \%$ (in C57N mice) of the total bacterial sequences. Other dominant phyla were Bacteroidetes, Proteobacteria and Deferribacteria ranging between $3 \%$ and 27\% (see Figure 1c). Moreover, in Figure 1d, a detailed overview of individual profiles of each mouse is illustrated on the phylum level (Figure 1d). In this study, the dominant phyla including Firmicutes, Proteobacteria and Deferribacteres showed significant differences between the groups (Figure 1c). Higher abundances of Firmicutes and Deferribacteres were observed in $\mathrm{C} 57 \mathrm{~N}$ mice and higher abundances of Proteobacteria in C57J mice. Bacteroidetes showed differences close to significance (adj. $P$-value $=0.052$ ) with higher abundances in C57J mice. No statistically significant differences were observed in less frequent phyla such as Tenericutes, TM7, Actinobacteria and Verrucomicrobia with abundances $<1 \%$ (summarized as 'others' in Figure 1c). Afterwards, we were focusing on differences on family level (Supplementary Table S1). The sequences of the most abundant OTUs, originated from each family were imported to ARB and matched against sequences of closest cultivated relatives. We observed differences in the phylum of Firmicutes that were represented by two families with higher abundances: Ruminococcaceae in C57N mice and Erysipelotrichaceae (summarized in Supplementary Table S2) in C57J mice. The most abundant OTU within Ruminococcaceae (C57): 6.4\%; C57N: 15.8\%, adj. $P$-value $\leqslant 0.05)$ had no cultured relative in the ARB rRNA sequence database, while the second most abundant OTU showed $95.9 \%$ identity to Anaerotruncus colihominis (C57J: 1.0\%; C57N: $3.1 \%$, adj. $P$-value $\leqslant 0.05)$. Within Erysipelotrichaceae, the most abundant OTU, which could be found mainly in C57J mice (C57J: $11.2 \%$; C57N: $0.001 \%$, adj. $P$-value $\leqslant 0.001$ ) was classified as Allobaculum, but was not closely related to any cultured organism (Allobaculum stercoricanis, $91.8 \%$ similarity). In addition, within the second most common phylum Bacteroidetes, the genus Bacteroides belonging to the Bacteroidaceae family was clearly more numerous in C57J mice (Supplementary Table S2), all identified as Bacteroides (B.) inhabiting mostly C57J mice (C57J: 12.5\%; C57N: $1.0 \%$, adj. $P$-value $\leqslant 0.05)$. The most abundant OTU showed $100 \%$ sequence similarity to next cultivated relatives $B$. fluxus and $99.5 \%$ to
B. uniformis and B. rodentium. Sequences belonging to the second most abundant OTU (C57J: 4.4\%; C57N: $0.3 \%$, adj. $P$-value $\leqslant 0.05)$ were $100 \%$ similar to $B$. acidifaciens and $B$. xylanisolvens. The differences between C57J and C57N mice in phylum Proteobacteria was limited to Epsilonproteobacteria. The highly abundant sequences in C57J mice were all classified to the family of Helicobacteraceae and belonged to the genus Helicobacter (H.). The most abundant OTU (C57J: 9.7\%; C57N: 0.7\%, adj. $P$-value $\leqslant 0.05)$ showed $100 \%$ sequence similarity to $H$. hepaticus and $97.9 \%$ similarity to $H$. bilis. The phylum Deferribacteres was composed of a single family, Deferribacteraceae, including Mucispirillum as highly abundant genus in C57N mice. The most abundant OTU (C57J: 2.5\%; C57N: 9.9\%, adj. $P$-value $\leqslant 0.05)$ was found to have $100 \%$ similarity to Mucispirillum schaedleri.

\section{Cecal meta-metabolomics reveals obesity-related} changes between the strains

We showed previously that the microbiome structure strongly influences the metabolite profiles by analysing human fecal water extracts in inflammatory bowel disease (Jansson et al., 2009). In this study, we followed the differences in the cecal but also the liver metabolome corresponding to the observed differences in the microbiome structure of the two strains. A MS-based metabolomics analysis of the cecal and liver samples was performed by direct infusion ESI FT-ICR-MS and complemented by ultra performance liquid chromatography time of flight MS/MS. To infer the metabolites associated with the body weight changes, we performed un- and supervised multivariate statistical analyses. The application of principal component analysis showed a sufficient separation between the groups in the first three dimensions, displaying the second and third components (Supplementary Figure S1A). In order to extrapolate the lists of mass signals causing the possible separation between the two classes (C57J vs C57N), an orthogonal partial least squares discriminant analysis (OPLS/O2PLS-DA) has been performed (Trygg, 2002; Trygg and Wold, 2002; Barker and Rayens, 2003; Bylesjö et al., 2006). The robustness of the model has been tested with cross-validation analysis of variance $(P=0.000275)$. The values of OPLS/O2PLS-DA model were 0.94 for goodness-of-fit $\mathrm{R}^{2} \mathrm{Y}(\mathrm{cum})$ and goodness-of-prediction of 0.78 for $\mathrm{Q}^{2}$ (cum). The scores scatter plot of the model is illustrated in Figure 2a. The mass signals responsible for the separation are highlighted in the corresponding S-plot, whereas some of the putative metabolites were illustrated (Figure 2b). Moreover, we examined the significance of the discriminative mass signals by applying a univariate statistical analysis. The application of non-parametric Wilcoxon-Mann-Whitney test resulted in 2453 significant mass signals (total $=10$ 515), whereas 488 were annotated in MassTRIX 
a

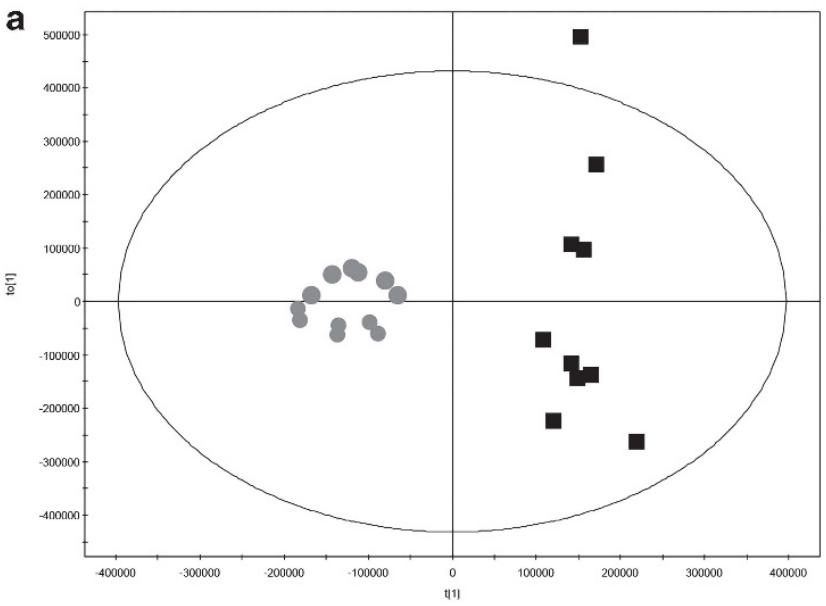

c
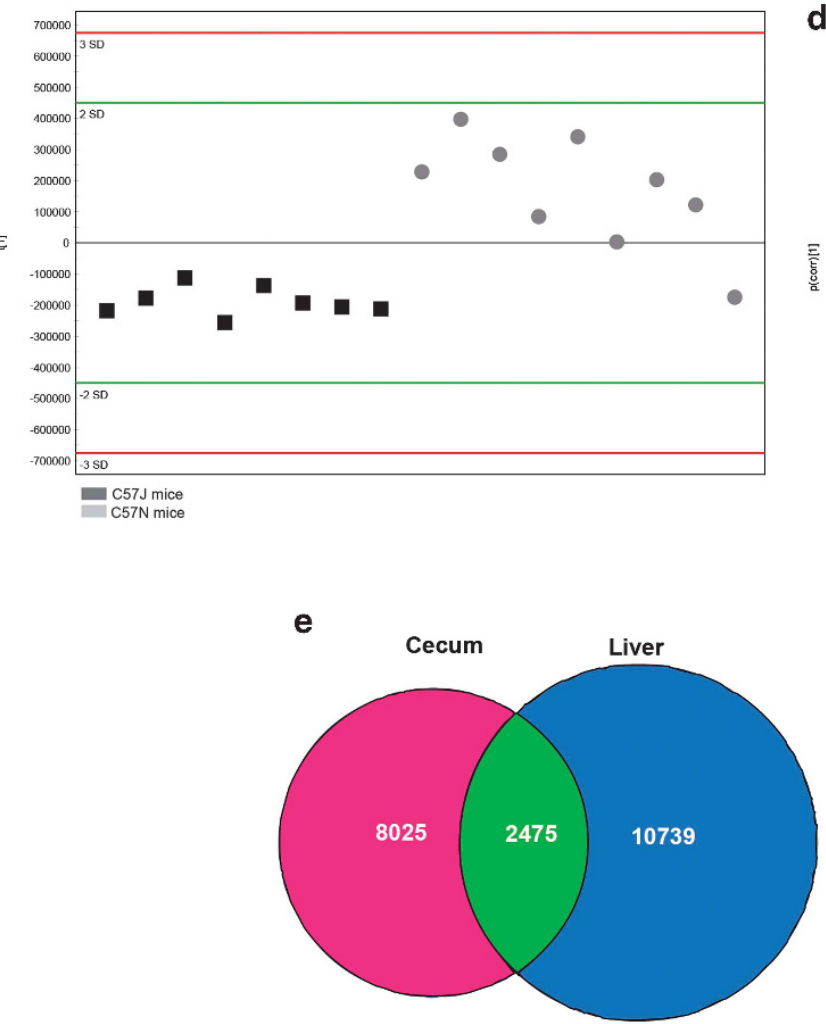

Total b
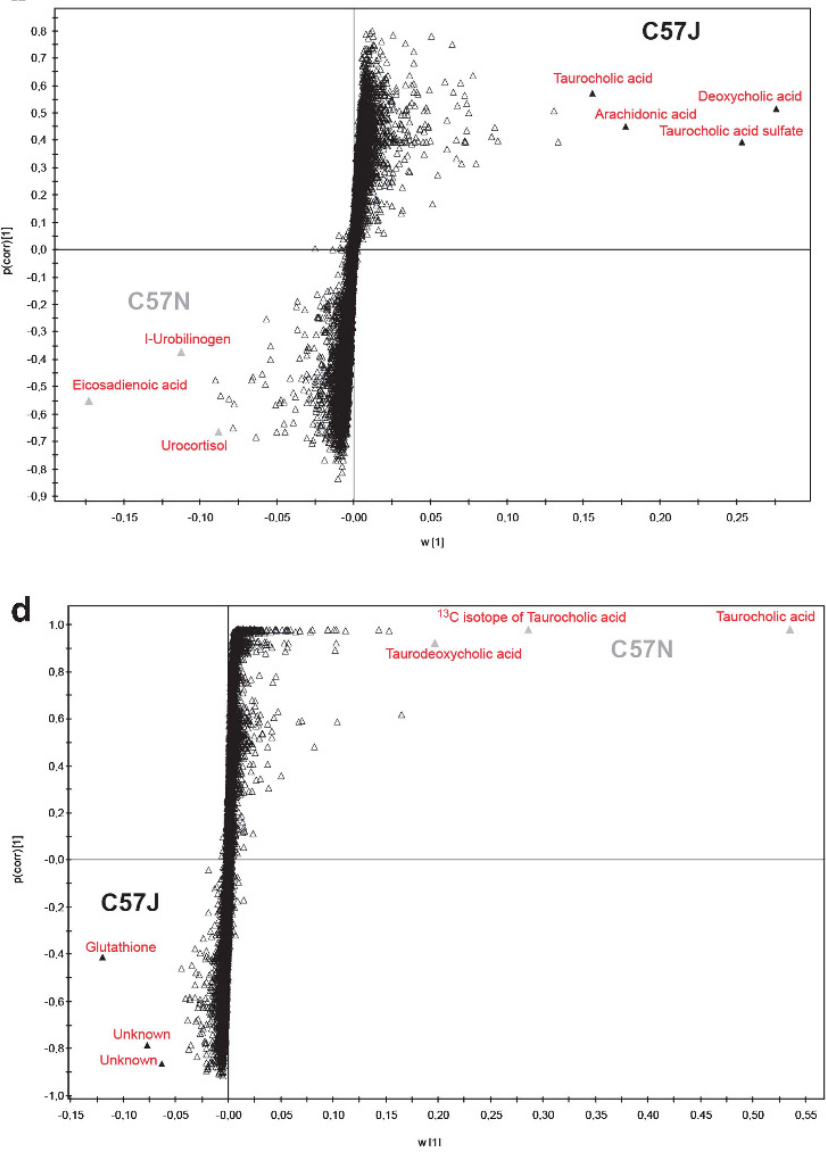

f

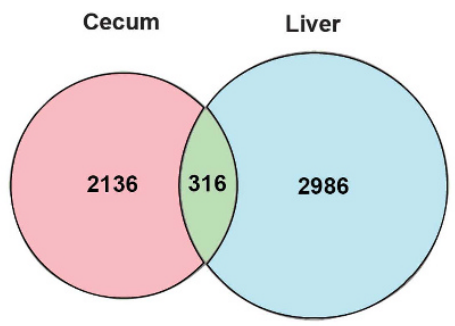

Significant

Figure 2 Non-targeted metabolomics performed with FT-ICR-MS reveals microbial and host related metabolome changes. (a) OPLS-DA scores scatter plot of cecal meta-metabolome (a) and liver metabolome (c) from C57J and C57N mice. (b) S-plot illustrated the putative metabolites responsible for the discrimination of C57J and C57N mice concerning the cecal (b) or liver metabolome (d). Venn diagram of total count of mass signals (e) detected commonly or uniquely in cecum or liver samples. Venn diagram of significant mass signals (f) detected commonly or uniquely in cecum or liver samples.

and remaining mass signals were defined as 'Unknowns', accented by an asterisk in all figures.

Liver metabolomics confirms the involvement of host metabolism due to obesity

To complement the host metabolome, we analysed extracted liver samples by FT-ICR-MS, followed by the same data processing steps applied before for the data matrix of cecal samples. In addition, here the application of unsupervised principal component analysis method for the liver metabolome resulted in a sufficient clustering of $\mathrm{C} 57 \mathrm{~J}$ and $\mathrm{C} 57 \mathrm{~N}$ mice (Supplementary Figure S1B). Besides, we subsequently performed an OPLS/O2PLS-DA, in order to classify and discriminate a possible group separation. We could find a valid model for the discrimination applying a cross-validation analysis 
of variance with a $P$-value smaller than 0.001 with the respective values of $\mathrm{R}^{2} \mathrm{Y}(\mathrm{cum})=0.67$ and $\mathrm{Q}^{2}(\mathrm{cum})=0.58$, with illustrated scores scatter plot in Figure 2c. Correspondingly, some of the putative metabolites are highlighted in the S-plot (Figure 2d). Here, the Wilcoxon-Mann-Whitney test resulted in 3327 significant mass signals and 470 mass signals could be annotated by MassTRIX (total=13 336). Subsequently, we compared the mass signals originated from both matrices in order to examine the similarity but also the uniqueness of both matrices by alignment within an error window of 1 p.p.m. In total, we found 21239 different mass signals, whereas 8025 mass signals were unique for cecal samples and 10379 were unique for liver samples (Figure 2e). About 2475 mass signals were matched in both matrices. Comparing only the significant mass signals, we found 5438 mass signals, whereas 316 were in common and 2986 uniquely significant in liver and 2136 in cecum (Figure 2f). Owing to the fact that in both comparisons the common signature is very low ( $11 \%$ in total and $5 \%$ in significant), we can conclude that cecal and liver metabolome are very unique and the metabolite patterns of cecum and liver changed differently and immensely between C57J and C57N mice.

Obesity affects a huge variety of metabolites in different classes detected in cecal meta-metabolome

The focus in this study was to discover the cecal meta-metabolome and how the body weight changes affects the metabolite patterns. According to the S-plot, we extrapolated eight mass signals, highlighted in Figure 2b, which were responsible for the discrimination of the achieved data analysis through OPLS/O2PLS-DA. The mass signals of the loadings are summarized in Supplementary Table S3. Four putative metabolites assigned as deoxycholic acid (DCA), taurocholic acid (TCA) sulfate, arachidonic acid and TCA characterized the group of C57J mice group, which was also confirmed by univariate statistics (Supplementary Table S3). Three other putative metabolites assigned as eicosadienoic acid, I-urobilinogen and urocortisol represented the group of $\mathrm{C} 57 \mathrm{~N}$ mice. This already indicates that specific metabolite classes such as fatty acids (FAs), bile acids (BAs), taurine-conjugated BAs (TBAs) and urobilinoids may have an essential role in discriminating the groups. Considering this information, we were looking specifically for these metabolite classes. We observed several metabolite classes of the BA metabolism such as $\mathrm{C}_{24} \mathrm{BAs}, \mathrm{C}_{24}$ TBAs, other conjugated $\mathrm{C}_{24} \mathrm{BAs}, \mathrm{C}_{27}$ TBAs and sulfates of $\mathrm{C}_{27}$ BAs. Moreover, several FAs and endocannabinoids were discriminative between C57J and C57N mice. Furthermore, bacterial metabolites were changed between C57J and C57N mice including urobilinoids and phenyl-containing metabolites.

\section{$B A$ metabolism}

In detail, we could find increased and significant different levels of oxolithocholic acid, oxocholenoic acid and cholandienoic acid in C57J mice, shown as a heatmap in Figure 3a. Increased but not significant patterns were observed for cholic acid and lithocholic acid. Contrary, other BAs such as hydroxycholic acid, trihydroxyoxocholanoic acid and trioxocholanoic acid were increased in C57N mice. Furthermore, we could detect $10 \mathrm{C}_{24}$ TBAs, shown in Figure 3b. Significantly increased TBAs (Tauro) were observed in C57J mice, including taurooxocholenoic acid, taurooxocholanoic acid, taurolithocholic acid (TDCA), taurodioxocholanoic acid, taurohydroxyoxocholanoic acid, taurodeoxycholic acid (TDCA), taurohydroxyoxocholanoic acid, TCA, taurohydroxycholanoic acid and the sulfate conjugate of TCA. Here we have to mention that six TBAs were not given in any database used for annotation. These unknown metabolites are highlighted by an asterisk in the heatmap (Figure 3).

Elevated levels were also measured for other conjugated $\mathrm{C}_{24}$ BAs such as glycodeoxycholic and glycoholic acid (Figure 3c). Among the $\mathrm{C}_{24}$ TBAs, we could find several $\mathrm{C}_{27}$ TBAs that were significantly increased in C57J mice, such as taurotrihydroxycholestanoic acid and taurodihydroxycholestanoic acid, which were annotated and already described in LIPID MAPS database. Moreover, we could find eight additional $\mathrm{C}_{27}$ TBAs, including taurodihydrocholestenoic acid, taurocholestenoic acid, taurodioxocholestanoic acid, taurodihydroxycholestenoic acid, taurodihydroxyoxocholestenoic acid, taurodihydroxycholestenoic acid, taurotetrahydroxycholestenoic acid and taurotetrahydroxycholestanoic acid, with increased ratios in C57J mice (Figure $3 \mathrm{~d}$ ). None of the eight $\mathrm{C}_{27}$ TBAs was given in the used metabolite databases. Finally, another class of sulfate conjugates of $\mathrm{C}_{27} \mathrm{BAs}$, including dihydroxyoxocholestanoic acid sulfate and dihydroxycholestenoic acid sulfate, increased in cecal samples of $\mathrm{C} 57 \mathrm{~N}$ mice, both belonging to 'Unknowns' (Figure 3e). Some $\mathrm{C}_{27}$ TBAs such as tauropenta-, taurotetra- and taurotrihydroxycholestanoic acid were already described and observed in urine of patients with Zellweger syndrome, Refsum's disease or with peroxisomes dysfunction in the liver (Lawson et al., 1986; Libert et al., 1991).

\section{Lipid metabolism}

As shown in the heatmap of Figure 3f, FAs were changed between C57J and C57N mice. Differences were observed in the ratios of arachidonic acid (C20:4), docosahexaenoic acid (C22:6), eicosapentaenoic acid (C20:5), retinoic acid, leukotriene B4, hydroxyl leukotriene B4 and hydroxyeicosatetraenoic acid with higher levels in C57J mice (Figure 3f). Contrarily, significantly decreased levels of eicosadienoic acid, docosadienoic acid (C20:2), docosatrienoic acid as well as pristanic acid were 


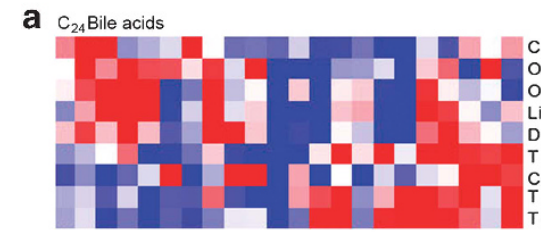

holandienoic acid xocholenoic acid xocholanoic acid thocholic acid eoxycholic acid rixoxocholanoic acid holic acid cholanoic acid etrahydroxycholanoic acid

b

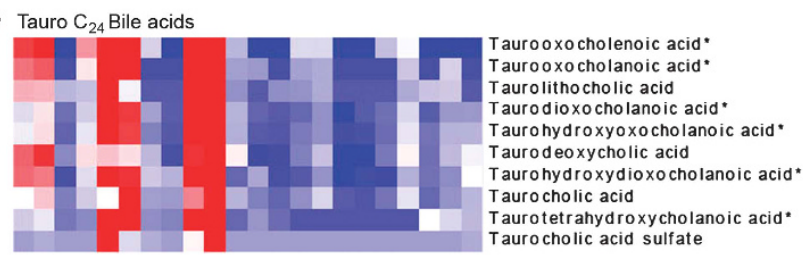

c

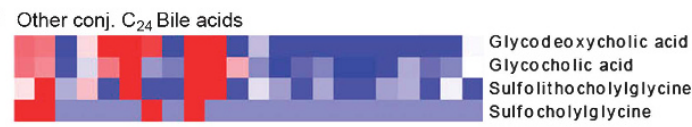

d Tauro $\mathrm{C}_{27}$ Bile acids

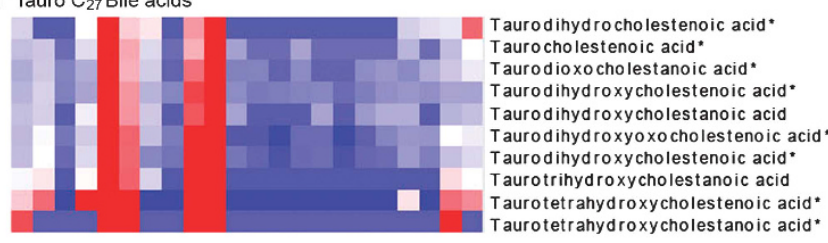

e Sulfates of $\mathrm{C}_{27}$ Bile acids

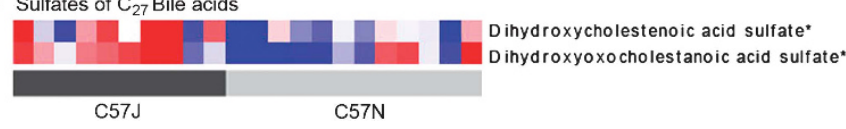

f Fatty acids

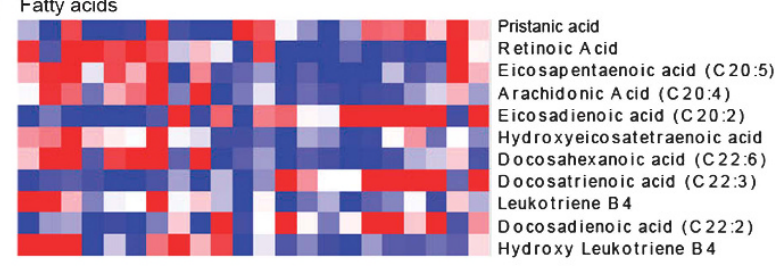

G Endocannabinoids

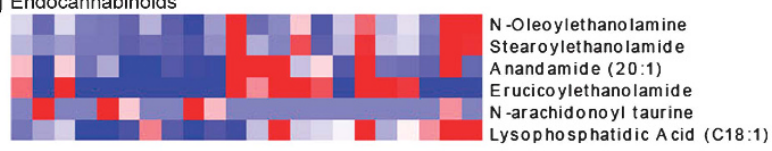

h Urobilinoids

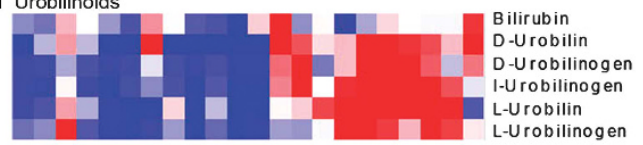

i Phenyl cont. metabolites

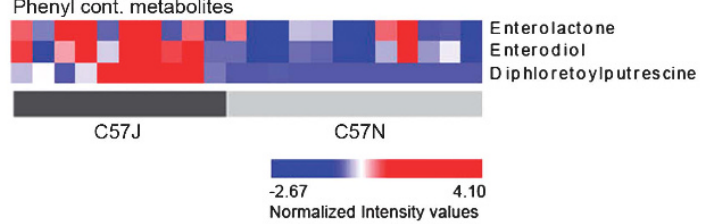

Figure 3 BA metabolism is influenced between C57J and C57N mice depicted through comparative analysis of cecal meta-metabolome. Obesity influences a variety of metabolite classes in cecum of C57J and C57N mice including $\mathrm{C}_{24} \mathrm{BAs}$ (a), tauro $\mathrm{C}_{24} \mathrm{BAs}$ (b), other conjugated BAs (c), tauro $\mathrm{C}_{27}$ BAs (d), sulfates of $\mathrm{C}_{27}$ BAs (e), FAs (f), endocannabinoids (g), urobilinoids (h) and phenyl-containing metabolites (i). *Unknown metabolites.

observed in C57J mice (Figure 3f). Interestingly, several endocannabinoid-like molecules were increased in $\mathrm{C} 57 \mathrm{~N}$ mice, primarily consisting of saturated/mono-unsaturated FAs (stearic acid, oleic acid (N-oleoylethanolamine; OEA), erucic acid and (C20:1) FA), shown in Figure 3g. Additionally, $\mathrm{N}$-arachidonoyltaurine and lysophosphatidic acid (C18:1) showed different patterns between C57J and C57N mice.

\section{Bacterial-derived metabolites}

Two main degradation metabolites of lignans, for example, secoisolariciresinol, the so-called enterolactone and enterodiol were increased in cecal content of C57J mice (Figure 3i). Besides the lignan catabolites, several bilirubin degradation compounds were increased in $\mathrm{C} 57 \mathrm{~N}$ mice (Figure $3 \mathrm{~h}$ ) including urobilinogens and urobilins such as D-, L- and I-urobilinogen and D- and L-urobilin. No changes were observed for bilirubin. Furthermore, we could identify a novel metabolite named diphloretoylputrescine (Figure 3i). All detailed information about the identification of the novel metabolite is summarized in Supplementary Information and the MS/MS is given in Supplementary
Figure S2A of Supplementary Information. The novel metabolite was significantly increased in cecal samples of C57J mice and was categorized into the class of polyamine conjugates. The most plausible structure is shown in Supplementary Figure S2B. Furthermore, MS/MS experiments performed by using ultra performance liquid chromatography time of flight MS/MS validate the presence of several metabolites as a representative of each class. We identified DCA, TDCA, arachidonic acid (C20:4), eicosadienoic acid (C20:2), enterolactone, L-urobilin, taurodihydroxycholestenoic acid and dihydroxyoxocholestanoic acid sulfate (Supplementary Figures S3A-H).

Liver metabolome underlines the effect of obesity but reveals opposite patterns of taurine-conjugated BAs As mentioned above, we could show that liver metabolome separates between C57J and C57N mice. Here, we elaborated only the metabolites that have already been described before. Moreover, we took only metabolites that were significant between C57J and $\mathrm{C} 57 \mathrm{~N}$ mice in liver samples. We detected metabolites involved in BAs metabolism, summarized in the heatmap of Figures $4 \mathrm{a}-\mathrm{e}$ and 
a

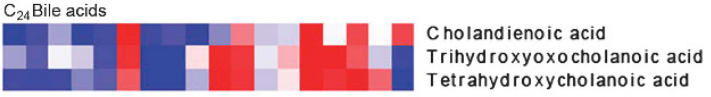

b

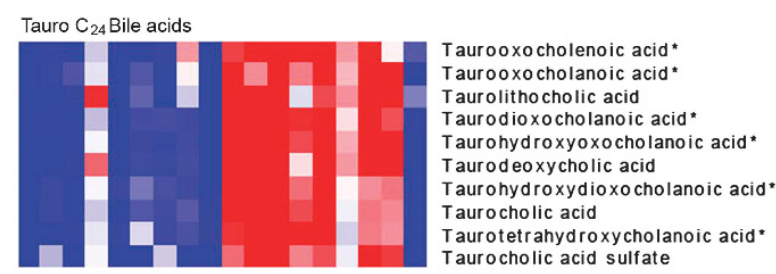

c

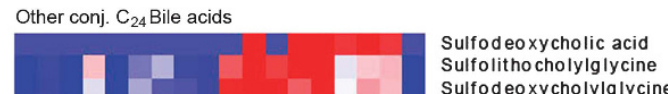

d

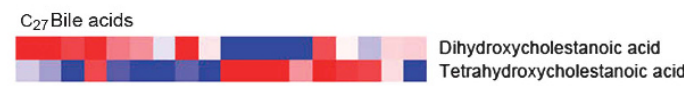

e Tauro $\mathrm{C}_{27}$ Bile acids

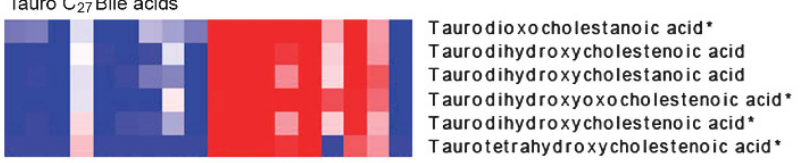

f

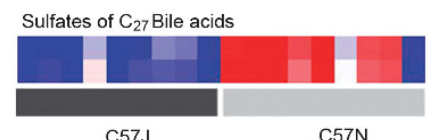

g

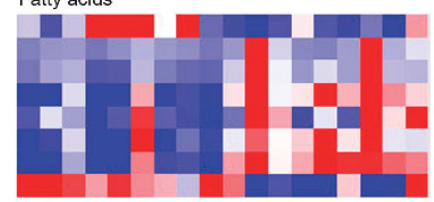

Retinoic A cid

Eicosapentaenoic acid (C 20:5) A rachidonic A cid (C20:4)

Eicosadienoic acid (C 20:2)

Docosahexanoic acid (C22:6)

Docosatrienoic acid (C 22:3)

Docosadienoic acid (C22:2)

Lysophosphatidic A cid (C18:1)

h

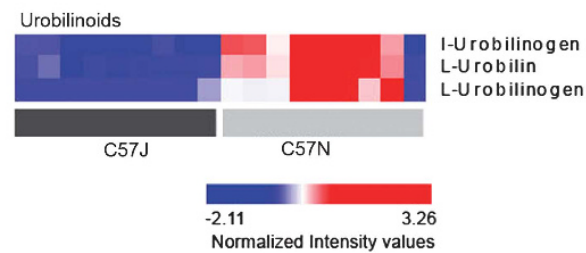

Figure 4 Liver metabolome reveals opposite patterns of conjugated BAs, especially $\mathrm{C}_{24}$ and $\mathrm{C}_{27}$ TBAs. Metabolite classes affected

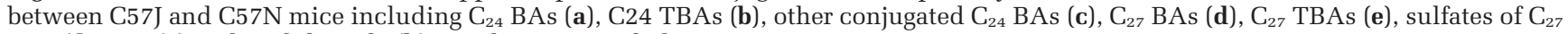
BAs (f), FAs (g) and urobilinoids (h). ${ }^{*}$ Unknown metabolites.

Supplementary Table S3. Especially the conjugated BAs such as $\mathrm{C}_{24}$ TBAs or $\mathrm{C}_{27}$ TBAs showed a complete opposite pattern between C57J and C57N mice, with elevated levels in C57N mice comparing with the cecal $\mathrm{C}_{24}$ and $\mathrm{C}_{27}$ TBAs that were elevated in C57J mice (Figure 3b). In addition, $\mathrm{C}_{27} \mathrm{BAs}$ such as di- and tetrahydroxycholestanoic acid were changed significantly in liver samples but not in cecal samples (Figure $4 \mathrm{~d}$ ). Accumulated levels of $\mathrm{C}_{27}$ BAs were already described in patients with mitochondrial dysfunction in Zellweger's syndrome (Hanson et al., 1979). Here all FAs were increased in the liver of C57N mice (Figure 4f). Lysophosphatidic acid (C18:1) and retinoic acid were increased in C57J mice. Urobilinoids showed similar patterns comparing with results of cecal meta-metabolome (Figure 4g). The phenyl-containing metabolites were not detected in liver samples in either C57J or C57N mice. Thus, we assume that these metabolites are exclusively derived from bacterial metabolism.

Impaired alpha oxidation in C57N mice depicted through involved metabolites

Especially, the accumulation of $\mathrm{C}_{27} \mathrm{BAS}$ and $\mathrm{C}_{27}$ TBAs in liver metabolome gives us a hint of possible dysfunction of liver peroxisomes in $\mathrm{C} 57 \mathrm{~N}$ mice because of appearance of unusual BAs, associated with impaired peroxisome function (Lawson et al., 1986; Libert et al., 1991). Perixosomal dysfunction was reported to have impaired alpha oxidation of phytanic acid (PA; Tien Poll-The et al., 1989). We detected higher levels of PA in liver samples of C57N mice, whereas no changes were shown for hydroxyPA and pristanic acid, shown in Figures 5a-c. Moreover, PA and hydroxyPA were not changed in cecal samples of $\mathrm{C} 57 \mathrm{~N}$ mice, but pristanic acid was elevated in $\mathrm{C} 57 \mathrm{~N}$ mice, shown in Figures $5 \mathrm{e}-\mathrm{g}$. Besides, phytol (Figure 5d) was also elevated significantly in cecum of C57N mice, but was not detected in liver samples.

\section{Dealing with 'Unknowns'-exploration of novel metabolites with mass difference analysis}

To unravel novel metabolites ('Unknowns'), we approached the experimental compositional space by annotation of all experimental mass signals through exact mass difference analysis using NetCalc and network visualization (Tziotis et al., 2011). Here, we used the data of the cecal metametabolome as an example, but this method is applicable for all mass spectra data derived from ultra-high-resolution MS such as FT-ICR-MS analysers. First, we calculated molecular formulas for all mass signals $(n=10515)$ within an error range of 0.2 p.p.m. Within this error range, we could find 5434 mass signals (pie diagram: red and black part, Figure 6a) with valid molecular formulas consisting of carbon, hydrogen, nitrogen, oxygen, 

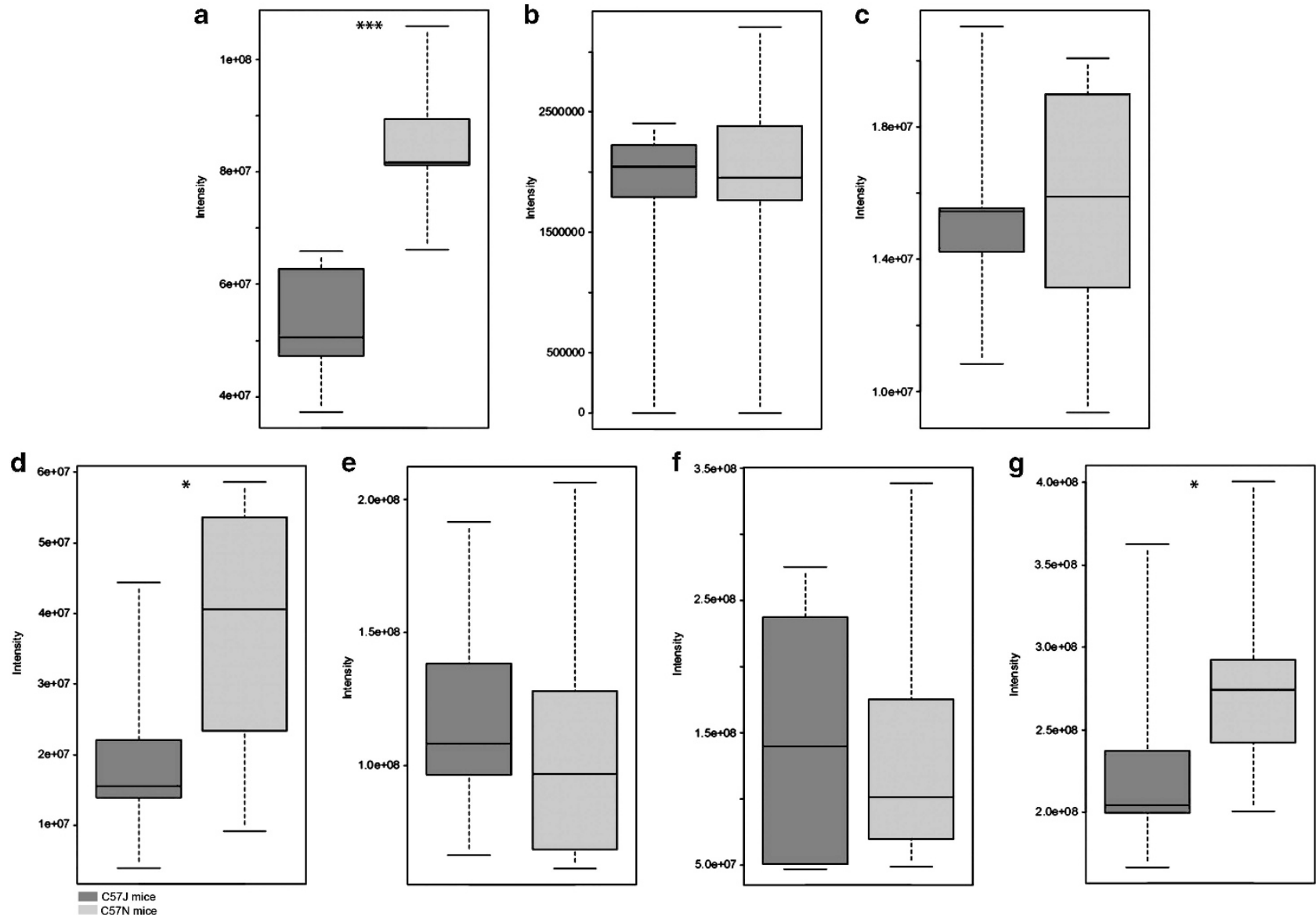

Figure 5 Impaired alpha-oxidation in C57N mice. C57N mice showed increased levels of PA (a), but no changes of hydroxyPA (b) or pristanic acid (c) in liver samples. Increased levels of phytol (d) were detected in cecal samples of C57N mice with no changes of PA (e), hydroxyPA (f) but elevated levels of pristanic acid (g). $P$-value: ${ }^{*}<0.05,{ }^{*}<0.01,{ }^{*}{ }^{*}<0.001$ (Wilcoxon-Mann-Whitney test).

sulfur and phosphorus, whereas 1438 mass signals were annotated in MassTRIX (black part, Figure 6a) and 3643 mass signals were solely assigned by their molecular formula (red part of the pie). There is still a huge remaining part of mass signals, which were not assigned by NetCalc (in total 5081 mass signals; gray part of the pie chart). Then, NetCalc performed the calculation of possible mass differences between the mass signals by applying a reference list consisting of 24 mass differences, for example, including biochemical transformations such as hydroxylation, taurine or sulfate conjugation (summarized in Supplementary Table S4, Supplementary Information). Afterwards, we were able to visualize the results of the mass difference analysis through a graphical representation of the generated network. The graph represents the metabolic network that consists of masses (nodes) with their respective molecular formula (Figure 6b, zoomed view in Figure 6c), edges (mass differences in gray color) and its connectivity, consisting of two main sub-graphs $\left({ }^{12} \mathrm{C}\right.$ and $\left.{ }^{13} \mathrm{C}\right)$. Moreover, the network is colored according the known (black nodes) and unknown (red nodes) mass signals with their molecular formula (blue labels), derived from the pie diagram. An example is displayed in Figure 6d, showing one node with the molecular formula of $\mathrm{C}_{20} \mathrm{H}_{32} \mathrm{O}_{5}$, which is putatively assigned as hydroxyl leukotriene B4 that possesses 23 connections to other nodes and belongs to one of the highly connected nodes of the total network. We could especially reveal new metabolites of hydroxyl leukotriene B4, connected by mass differences with S-containing molecular formulas, shown in Figure 6d.

\section{Discussion}

In our study, we showed that body weight gain, observed in one of two different mouse strains after HFD feeding, resulted in diverse gut microbiome and metabolome patterns. To unravel the complex gut microbial ecology, we applied and combined two high-resolution techniques, that is, 454 pyrosequencing and FT-ICR-MS-based metabolomics. The application of HFD for 3 weeks led to strong body weight changes particularly in the $\mathrm{C} 57 \mathrm{~N}$ strain with different microbial or host-related metabolome patterns. In this study, we detect several phyla and families of the gut microbiome that were also observed in human, mouse or other animal studies 

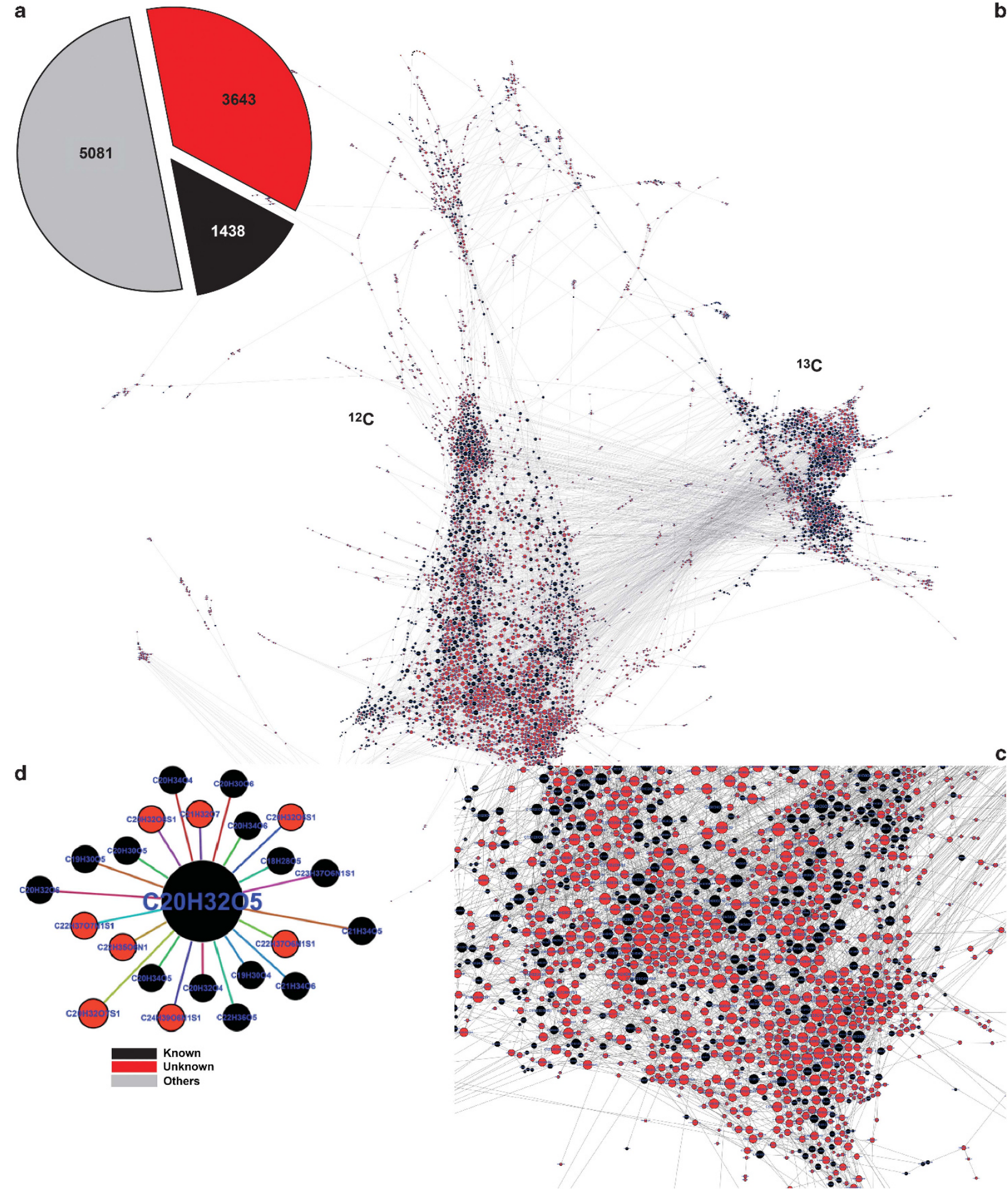

Figure 6 Mass difference network analysis and visualization-exploration of 'Unknowns'. (a) Pie diagram illustrating the number of total mass signals detected in cecal meta-metabolome data set of (-) FT-ICR-MS (total =10 515 mass signals); consisting of 5434 mass signals that were assigned to molecular formulas with carbon, hydrogen, nitrogen, oxygen, sulfur and phosphorus (CHNOSP) composition, divided into Unknown mass signals (red) and known metabolites (black), which were annotated in MassTRIX. The gray part of the pie diagram consists of mass signals (5081) that were not assigned after NetCalc annotation. A mass difference network is illustrated in b generated from 5434 mass signals (nodes, black and red nodes) and 24 mass differences (edges) are colored in gray. Detailed inspection of the network in c; (d) A mass signal with molecular formula of $\mathrm{C}_{20} \mathrm{H}_{32} \mathrm{O}_{5}$, assigned as hydroxyl leukotriene B4, possessing edges to known and unknown mass signals. 
as common gut flora or during HFD exposure in different metabolic phenotypes (Zhang et al., 2010; Kim et al., 2012b). The change of the bacterial community composition, especially for Firmicutes/ Bacteroidetes ratio, with enriched abundances of Firmicutes and depleted occurrence of Bacteroidetes in the obese C57N phenotype is in accordance with many former studies (Ley et al., 2005; Turnbaugh et al., 2006, 2009; Cani et al., 2007). It has been postulated that HFD is one of the factor responsible for changes in the bacterial community. Bacteria are able to harvest more energy from dietary nutrients (Turnbaugh et al., 2006), likely resulting in higher body weight changes in the C57N strain. In another mouse study, Erysipelotrichaceae were reported to be the predominant family (Zhang et al., 2010). Here we demonstrated that in C57N mice this family was suppressed, while Ruminococcaceae were present in increased abundances compared to lean C57J mice. Higher abundances of Ruminococcaceae are in accordance with other studies following HFD intervention (Zhang et al., 2010; Kim et al., 2012b). The observed appearance of $B$. uniformis was reported before, whereas oral administration of a $B$. uniformis strain counteracts HF feeding and was able to reduce body weight gain, liver steatosis and reduce dietary fat absorption (Gauffin Cano et al., 2012). However, the observation of decreased abundance of Proteobacteria after HFD application in C57N mice is contradictory to previous results (Hildebrandt et al., 2009; Geurts et al., 2011). The third highest abundant OTU in gut bacterial community of C57J mice was highly similar to $H$. hepaticus and to a lower degree similar to $H$. bilis. Both are known to colonize the intestinal mucus and have been found in liver, in the latter case even in bile (Fox et al., 1995, 2011). As a consequence of HFD-induced obesity, the abundance of phylum Deferribacteres increased in the gut microbiome, especially the abundance of the genus Mucispirillum (Ravussin et al., 2012; Serino et al., 2012). The highly abundant OTU belonging to Mucispirillum schaedleri seems to be an important factor in the metabolism of C57N mice. It is known to colonize the mucus layer, has been found in ileal, colonic and liver tissue samples and is assumed to be capable of translocating from the intestinal tract to the hepatobilary system (Robertson et al., 2005). As no earlier studies have focused on the differences between the gut microbiota between these mouse strains, our results reveal interesting aspects of bacteria that could be involved in obesity under the impact of HFD. The uniqueness of the study lies in the focus on two mouse strains expressing small variations at the genetic level but strong variations in HFD-induced obesity.

Concerning the metabolome, until now several metabolomics studies have been performed to unravel the microbial metabolism following different biological questions. Many of these studies investigated the particular role of metabolites in inflammatory bowel diseases by using primarily NMR studies (Lin et al., 2011). Non-targeted metabolomics approaches in gut microbial sample matrices and liver, analysing changes occurring in metabolic diseases like obesity, are rarely given, but many studies addressed obesity-related metabolome characterization (Dumas et al., 2006; Williams et al., 2006; Fearnside et al., 2008; Li et al., 2008, 2010a; Shearer et al., 2008; Newgard et al., 2009; Waldram et al., 2009; Kim et al., 2009, 2010, 2011; Calvani et al., 2010; Xie et al., 2010, 2012; Zhao et al., 2010; Oberbach et al., 2011; Duggan et al., 2011a,b; Jung et al., 2012; Hanhineva et al., 2013; Schäfer et al., 2014; Seyfried et al., 2013; Won et al., 2013; Xu et al., 2013; Daniel et al., 2014; Eisinger et al., 2014). Comparing with other studies, our study provides a greater insight into different metabolite classes that were involved in obesity-related changes by reflecting both bacterial and host metabolism. Here, we highlighted in comprehensive manner the metabolome changes in the complex environment of the gut microbiome, given in both strains and compared them to the liver metabolome. Both organ systems were immensely altered between the strains and several metabolite classes were shown to be differentially affected between C57J and C57N mice. A major imbalance occurred in BA metabolism of cecum and liver, revealing distinct profiles of free and conjugated BAs. FAs, endocannabinoids, urobilinoids as metabolites of the bilirubin degradation pathway and metabolites of lignans, enterodiol and enterolactone are reflecting different bacterial and liver metabolism. The BA metabolism is one of the important key factors in obesity-related changes, including free and conjugated BAs. BAs are known to exert several biological effects in vivo, such as having a role in lipid and cholesterol metabolism but also act as signaling molecules activating nuclear hormone receptors, affecting body weight and insulin resistance (Watanabe et al., 2006; Lefebvre et al., 2009; Li et al., 2010b). Distinct BA levels in cecal samples could be derived through altered enterohepatic circulation, bacterial metabolism or increased BA excretion. As one example, DCA is one of the major secondary BAs derived through dehydroxylation of bacteria in the gut. Narushima et al. (2006) indicate that Bacteroides is the main bacterial genus that is responsible for the dehydroxylation of CA to DCA. In our study, elevated BAs and simultaneously higher abundances of $B$. acidifaciens could be observed in the C57J strain, indicating that co-occurrence of elevated BAs and $B$. acidifaciens could provide a protection against HFD-induced obesity in C57J mice. Miyamoto and Itoh (2000) discovered $B$. acidifaciens in murine cecum and described its growth capability in high contents of bile. Interestingly, elevated BA was observed in feces of diabetic mice (ob/ob mice) with elevated levels of CA and DCA (Li et al., 2012). Thus, it can be explained through different regulation of BA pool, which is 
depending on the disease state. Transgenic mice overexpressing cholesterol $7 \alpha$-hydroxylase, which is involved in the biosynthesis of BAs from cholesterol, were resistant against diet-induced weight gain and development of fatty liver with elevated BAs concentration in the intestine (Li et al., 2010b). Administration of CA to obese C57J mice that were fed with HFD led to a decrease of body weight and improves insulin resistance. This effect was also observed in KK-Ay mice, which showed increased levels of CA, DCA, TCA and TDCA in liver and intestine, which were also observed in our study in cecal samples of C57J mice fed with a HFD (Watanabe et al., 2006). The patterns of TBAs can be modulated through diet, absence of bacteria, distinct microbiome community, BAs or farnesoid X receptors agonists (Claus et al., 2008; Li et al., 2010a; Swann et al., 2011; Watanabe et al., 2011). In this study, lower abundance of TBAs in cecum of C57N mice could also be explained through an increase in their deconjugation rate by specific gut bacteria that are using the sulfur-containing taurine as an energy source such as Enterobacteria or Bacteroides spp. (Ridlon et al., 2006; Martin et al., 2007). Moreover, the prominent opposite levels of all TBAs, comparing cecal and liver system, underlines a possible bacterial involvement. The detection of several $\mathrm{C}_{27}$ TBAs indicated a possible liver peroxisome dysfunction in $\mathrm{C} 57 \mathrm{~N}$ mice, affirmed through metabolites of impaired alpha oxidation (Steinberg et al., 2006). Especially, FAs that were increased in C57J mice, are important modulators of peroxisome proliferator-activated receptors activity, involved in FA and lipid homeostasis (Krey et al., 1997). Here, elevated FAs in liver of C57N mice and decreased FAs in cecal samples are probably due to decreased excretion. Endocannabinoid-like metabolites such as OEA and lysophosphatidic acid were elevated in cecum of C57N mice and lysophosphatidic acid was lower in liver samples of obese C57N mice. OEA is one of the endocannabinoid-like metabolites, which is modulating satiety and decreases body weight through activation of peroxisome proliferatoractivated receptor- $\alpha$ but do not regulate the endocannabionid system (Capasso and Izzo, 2008). Lysophosphatidic acid is another bioactive lipid that mediates adipocyte proliferation and differentiation through downregulation of peroxisome proliferator-activated receptor- $\gamma$ and is involved in obesity (Nobusue et al., 2010; Federico et al., 2012). Enterolactone and enterodiol are bacterial metabolites, produced by certain bacteria and have been reported to exert beneficial effects in the body (Woting et al., 2010). Studies showed that low concentrations of enterolactone in human plasma were positively correlated with obesity, which are similar to our results (Sonestedt et al., 2008). Obese C57N mice showed also lower levels of enterolactone in cecum. Subcutaneous injection of enterolactone and enteridiol tend to decrease body weight in mice (Tominaga et al., 2012). The patterns of urobilinoids are likely due to different communities that were observed in C57J and C57N mice by our 16S-rRNA gene analyses. The degradation of bilirubin occurs through intestinal bacteria such as Clostridium spp. (Tiribelli and Ostrow, 2005). However, also one further phenolic compound seems to have an important role in discriminating C57J and C57N mice. Especially, a conjugate of two molecules of phloretic acid and putrescine, named diphloretoylputrescine is assumed to be an important metabolite in C57J mice. Moreover, this metabolite was only detected in the cecum, assuming to be derived through bacterial metabolism. Phloretic acid is a degradation product of the tyrosine metabolism and putrescine is derived through bacterial degradation of proteins (Booth et al., 1960). Previously related, so-called polyamine conjugates with different phenol-containing molecules such as coumaric, ferulic and caffeic acid were discovered, predominantly in plants (Moreau et al., 2001; Choi et al., 2007). Especially, the conjugates dicoumaroylputrescine and diferuoylputrescine were shown to be anti-inflammatory and inhibit nitric oxide production in macrophages (Kim et al., 2012a).

Our study clearly discriminates between C57J and $\mathrm{C} 57 \mathrm{~N}$ mice under HFD conditions and shows changes of the cecal microbiome and metabolome reflecting alterations in the gut bacterial community composition and metabolism of host and microbiome. Applying both approaches, we were able to access directly some functional aspects in the host-gut microbiome interactions and metabolism and identify new factors that could contribute to HFD-induced obesity. A deeper understanding of possible triggers for these dysbalance patterns could also provide a new strategy for engineering the gut microbiome, to counteract body weight gain and to treat obesity.

\section{Conflict of Interest}

The authors declare no conflict of interest.

\section{Acknowledgements}

This work was supported and funded by German Center for Diabetes Research.

\section{References}

Antunes LCM, Han J, Ferreira RBR, Lolić P, Borchers CH, Finlay BB. (2011). Effect of antibiotic treatment on the intestinal metabolome. Antimicrob Agents Chemother 55: 1494-1503.

Barker M, Rayens W. (2003). Partial least squares for discrimination. J Chemometrics 17: 166-173.

Baur P, Martin F-P, Gruber L, Bosco N, Brahmbhatt V, Collino $S$ et al. (2011). Metabolic phenotyping of the Crohn's disease-like IBD etiopathology in the TNF $\triangle$ ARE/WT mouse model. J Proteome Res 10: 5523-5535. 
Benjamini Y, Hochberg Y. (1995). Controlling the false discovery rate: a practical and powerful approach to multiple testing. J R Stat Soc B 289: 300-1188.

Booth AN, Masri MS, Robbins DJ, Emerson OH, Jones FT, DeEds F. (1960). Urinary phenolic acid metabolites of tyrosine. J Biol Chem 235: 2649-2652.

Bylesjö M, Rantalainen M, Cloarec O, Nicholson JK, Holmes E, Trygg J. (2006). OPLS discriminant analysis: combining the strengths of PLS-DA and SIMCA classification. J Chemometrics 20: 341-351.

Bäckhed F, Ding H, Wang T, Hooper LV, Koh GY, Nagy A et al. (2004). The gut microbiota as an environmental factor that regulates fat storage. Proc Natl Acad Sci USA 101: 15718-15723.

Bäckhed F, Manchester JK, Semenkovich CF, Gordon JI. (2007). Mechanisms underlying the resistance to diet-induced obesity in germ-free mice. Proc Natl Acad Sci USA 104: 979-984.

Caballero B. (2007). The global epidemic of obesity: an overview. Epidemiol Rev 29: 1-5.

Calvani R, Miccheli A, Capuani G, Miccheli AT, Puccetti C, Delfini $M$ et al. (2010). Gut microbiome-derived metabolites characterize a peculiar obese urinary metabotype. Int J Obes 34: 1095-1098.

Cani PD, Amar J, Iglesias MA, Poggi M, Knauf C, Bastelica D et al. (2007). Metabolic endotoxemia initiates obesity and insulin resistance. Diabetes 56: 1761-1772.

Capasso R, Izzo AA. (2008). Gastrointestinal regulation of food intake: general aspects and focus on anandamide and oleoylethanolamide. J Neuroendocrinol 20: 39-46.

Choi SW, Lee SK, Kim EO, Oh JH, Yoon KS, Parris N et al. (2007). Antioxidant and antimelanogenic activities of polyamine conjugates from corn bran and related hydroxycinnamic acids. J Agric Food Chem 55: 3920-3925.

Claus SP, Tsang TM, Wang Y, Cloarec O, Skordi E, Martin F-P et al. (2008). Systemic multicompartmental effects of the gut microbiome on mouse metabolic phenotypes. Mol Syst Biol 4: 219.

Cole JR, Wang Q, Cardenas E, Fish J, Chai B, Farris RJ et al. (2009). The ribosomal database project: improved alignments and new tools for rRNA analysis. Nucleic Acids Res 37: D141-D145.

Daniel H, Gholami AM, Berry D, Desmarchelier C, Hahne H, Loh G et al. (2014). High-fat diet alters gut microbiota physiology in mice. ISME J 8: 295-308.

David LA, Maurice CF, Carmody RN, Gootenberg DB, Button JE, Wolfe BE et al. (2013). Diet rapidly and reproducibly alters the human gut microbiome. Nature 505: $559-563$.

Dixon P. (2003). VEGAN, a package of $\mathrm{R}$ functions for community ecology. J Veg Sci 14: 927-930.

Dray S, Dufour AB. (2007). The ade4 package: implementing the duality diagram for ecologists. J Stat Softw 22: 1-20.

Duggan GE, Hittel DS, Hughey CC, Weljie A, Vogel HJ, Shearer J. (2011a). Differentiating short- and long-term effects of diet in the obese mouse using $1 \mathrm{H}$-nuclear magnetic resonance metabolomics. Diabetes Obes Metab 13: 859-862.

Duggan GE, Hittel DS, Sensen CW, Weljie AM, Vogel HJ, Shearer J. (2011b). Metabolomic response to exercise training in lean and diet-induced obese mice. J Appl Physiol (Bethesda, MD: 1985) 110: 1311-1318.

Dumas ME, Barton RH, Toye A, Cloarec O, Blancher C, Rothwell A et al. (2006). Metabolic profiling reveals a contribution of gut microbiota to fatty liver phenotype in insulin-resistant mice. Proc Natl Acad Sci USA 103: 12511-12516.

Edgar RC, Haas BJ, Clemente JC, Quince C, Knight R. (2011). UCHIME improves sensitivity and speed of chimera detection. Bioinformatics 27: 2194-2200.

Eisinger K, Liebisch G, Schmitz G, Aslanidis C, Krautbauer S, Buechler C. (2014). Lipidomic analysis of serum from high fat diet induced obese mice. Int J Mol Sci 15: 2991-3002.

Fearnside JF, Dumas M-E, Rothwell AR, Wilder SP, Cloarec O, Toye A et al. (2008). Phylometabonomic patterns of adaptation to high fat diet feeding in inbred mice. PLoS One 3: e1668.

Federico L, Ren H, Mueller Pa WuT, Liu S, Popovic J et al. (2012). Autotaxin and its product lysophosphatidic acid suppress brown adipose differentiation and promote diet-induced obesity in mice. Mol Endocrinol (Baltimore, MD) 26: 786-797.

Fox JG, Ge Z, Whary MT, Erdman SE, Horwitz BH. (2011). Helicobacter hepaticus infection in mice: models for understanding lower bowel inflammation and cancer. Mucosal Immunol 4: 22-30.

Fox JG, Yan LL, Dewhirst FE, Paster BJ, Shames B, Murphy JC et al. (1995). Helicobacter bilis sp. nov., a novel Helicobacter species isolated from bile, livers, and intestines of aged, inbred mice. J Clin Microbiol 33: $445-454$.

Gauffin Cano P, Santacruz A, Moya Á, Sanz Y. (2012). Bacteroides uniformis CECT 7771 ameliorates metabolic and immunological dysfunction in mice with high-fat-diet induced obesity. PLoS One 7: e41079.

Geurts L, Lazarevic V, Derrien M, Everard A, Van Roye M, Knauf $\mathrm{C}$ et al. (2011). Altered gut microbiota and endocannabinoid system tone in obese and diabetic leptin-resistant mice: impact on apelin regulation in adipose tissue. Front Microbiol 2: 149.

Hanhineva K, Barri T, Kolehmainen M, Pekkinen J, Pihlajamäki J, Vesterbacka A et al. (2013). Comparative nontargeted profiling of metabolic changes in tissues and biofluids in high-fat diet-fed Ossabaw pig. J Proteome Res 12: 3980-3992.

Hanson RF, Szczepanik-VanLeeuwen P, Williams GC, Grabowski G, Sharp HL. (1979). Defects of bile acid synthesis in Zellweger's syndrome. Science 203: 1107-1108.

Hildebrandt MA, Hoffmann C, Sherrill-Mix SA, Keilbaugh SA, Hamady M, Chen YY et al. (2009). High-fat diet determines the composition of the murine gut microbiome independently of obesity. Gastroenterology 137: 1716-1724.

Jansson J, Willing B, Lucio M, Fekete A, Dicksved J, Halfvarson J et al. (2009). Metabolomics reveals metabolic biomarkers of Crohn's disease. PLoS One 4: e6386.

Jung JY, Kim IY, Kim YN, Kim JS, Shin JH, Jang ZH et al. (2012). 1H NMR-based metabolite profiling of dietinduced obesity in a mouse mode. BMB Rep 45: 419-424.

Juretschko S, Timmermann G, Schmid M, Schleifer K-H, Pommerening-Röser A, Koops H-P et al. (1998). Combined molecular and conventional analyses of nitrifying bacterium diversity in activated sludge: Nitrosococcus mobilis and Nitrospira-like bacteria as dominant populations. Appl Environ Microbiol 64: 3042-3051.

Kahn BB, Flier JS. (2000). Obesity and insulin resistance. J Clin Invest 106: 473-481. 
Kim EO, Min KJ, Kwon TK, Um BH, Moreau RA, Choi SW. (2012a). Anti-inflammatory activity of hydroxycinnamic acid derivatives isolated from corn bran in lipopolysaccharide-stimulated Raw 264.7 macrophages. Food Chem Toxicol 50: 1309-1316.

Kim HJ, Kim JH, Noh S, Hur HJ, Sung MJ, Hwang JT et al. (2011). Metabolomic analysis of livers and serum from high-fat diet induced obese mice. J Proteome Res 10: 722-731.

Kim JY, Park JY, Kim OY, Ham BM, Kim H-J, Kwon DY et al. (2010). Metabolic profiling of plasma in overweight/ obese and lean men using ultra performance liquid chromatography and Q-TOF mass spectrometry (UPLC - Q-TOF MS). J Proteome Res 9: 4368-4375.

Kim K-A, Gu W, Lee I-A, Joh E-H, Kim D-H. (2012b). High fat diet-induced gut microbiota exacerbates inflammation and obesity in mice via the TLR4 signaling pathway. PLoS One 7: e47713.

Kim S-H, Yang S-O, Kim H-S, Kim Y, Park T, Choi H-K. (2009). 1H-nuclear magnetic resonance spectroscopybased metabolic assessment in a rat model of obesity induced by a high-fat diet. Anal Bioanal Chem 395: 1117-1124.

Krey G, Braissant O, L'Horset F, Kalkhoven E, Perroud M, Parker MG et al. (1997). Fatty acids, eicosanoids, and hypolipidemic agents identified as ligands of peroxisome proliferator-activated receptors by coactivatordependent receptor ligand assay. Mol Endocrinol (Baltimore, MD) 11: 779-791.

Lane D. (1991). 16S/23S rRNA sequencing. In: Stackebrandt E, Goodfellow M (eds) Nucleic Acid Techniques in Bacterial Systematics, 1st edn. Wiley: New York, pp 115-175.

Lawson AM, Madigan MJ, Shortland D, Clayton PT. (1986). Rapid diagnosis of Zellweger syndrome and infantile Refsum's disease by fast atom bombardment-mass spectrometry of urine bile salts. Clinica Chimica Acta 161: 221-231.

Lefebvre P, Cariou B, Lien F, Kuipers F, Staels B. (2009). Role of bile acids and bile acid receptors in metabolic regulation. Physiol Rev 89: 147-191.

Ley RE, Bäckhed F, Turnbaugh P, Lozupone Ca, Knight RD, Gordon JI. (2005). Obesity alters gut microbial ecology. Proc Natl Acad Sci USA 102: 11070-11075.

Li H, Xie Z, Lin J, Song H, Wang Q, Wang K et al. (2008). Transcriptomic and metabonomic profiling of obesityprone and obesity-resistant rats under high fat diet. J Proteome Res 7: 4775-4783.

Li LO, Hu YF, Wang L, Mitchell M, Berger A, Coleman RA. (2010a). Early hepatic insulin resistance in mice: a metabolomics analysis. Mol Endocrinol (Baltimore, MD) 24: 657-666.

Li T, Francl JM, Boehme S, Ochoa A, Zhang Y, Klaassen CD et al. (2012). Glucose and insulin induction of bile acid synthesis: mechanisms and implication in diabetes and obesity. J Biol Chem 287: 1861-1873.

Li T, Owsley E, Matozel M, Hsu P, Novak CM, Chiang JYL. (2010b). Transgenic expression of cholesterol 7alpha-hydroxylase in the liver prevents high-fat diet-induced obesity and insulin resistance in mice. Hepatology (Baltimore, MD) 52: 678-690.

Libert R, Hermans D, Draye JP, Van Hoof F, Sokal E, de Hoffmann E. (1991). Bile acids and conjugates identified in metabolic disorders by fast atom bombardment and tandem mass spectrometry. Clin Chem 37: 2102-2110.
Lin H-M, Helsby NA, Rowan DD, Ferguson LR. (2011). Using metabolomic analysis to understand inflammatory bowel diseases. Inflammatory Bowel Diseases 17: 1021-1029.

Lucio M, Fekete A, Weigert C, Wägele B, Zhao X, Chen J et al. (2010). Insulin sensitivity is reflected by characteristic metabolic fingerprints-a fourier transform mass spectrometric non-targeted metabolomics approach. PLoS One 5: e13317.

Ludwig W, Strunk O, Westram R, Richter L, Meier H, Yadhukumar et al. (2004). ARB: a software environment for sequence data. Nucleic Acids Res 32: 1363-1371.

Martin F-PJ, Dumas M-E, Wang Y, Legido-Quigley C, Yap IKS, Tang $\mathrm{H}$ et al. (2007). A top-down systems biology view of microbiome-mammalian metabolic interactions in a mouse model. Mol Syst Biol 3: 112.

Matsumoto M, Kibe R, Ooga T, Aiba Y, Kurihara S, Sawaki E et al. (2012). Impact of intestinal microbiota on intestinal luminal metabolome. Sci Rep 2: 233.

Mekada K, Abe K, Murakami A, Nakamura S, Nakata H, Moriwaki K et al. (2009). Genetic differences among C57BL/6 substrains. Exp Anim 58: 141-149.

Miyamoto Y, Itoh K. (2000). Bacteroides acidifaciens sp, nov., isolated from the caecum of mice. Int J Syst Evol Microbiol 50: 145-148.

Montgomery MK, Hallahan NL, Brown SH, Liu M, Mitchell TW, Cooney GJ et al. (2013). Mouse straindependent variation in obesity and glucose homeostasis in response to high-fat feeding. Diabetologia 56: 1129-1139.

Moreau R, Nuñez A, Singh V. (2001). Diferuloylputrescine and p-coumaroyl-feruloylputrescine, abundant polyamine conjugates in lipid extracts of maize kernels. Lipids 36: 839-844.

Narushima S, Itoha K, Miyamoto Y, Park S-H, Nagata K, Kuruma K et al. (2006). Deoxycholic acid formation in gnotobiotic mice associated with human intestinal bacteria. Lipids 41: 835-843.

Neish AS. (2009). Microbes in gastrointestinal health and disease. Gastroenterology 136: 65-80.

Newgard CB, An J, Bain JR, Muehlbauer MJ, Stevens RD, Lien LF et al. (2009). A branched-chain amino acid-related metabolic signature that differentiates obese and lean humans and contributes to insulin resistance. Cell Metab 9: 311-326.

Nicholson A, Reifsnyder PC, Malcolm RD, Lucas CA, MacGregor GR, Zhang W et al. (2010). Diet-induced obesity in two C57BL/6 substrains with intact or mutant nicotinamide nucleotide transhydrogenase (Nnt) gene. Obesity 18: 1902-1905.

Nicholson JK, Lindon JC, Holmes E. (1999). 'Metabonomics': understanding the metabolic responses of living systems to pathophysiological stimuli via multivariate statistical analysis of biological NMR spectroscopic data. Xenobiotica 29: 1181-1189.

Nobusue H, Kondo D, Yamamoto M, Kano K. (2010). Effects of lysophosphatidic acid on the in vitro proliferation and differentiation of a novel porcine preadipocyte cell line. Comp Biochem Physiol B Biochem Mol Biol 157: 401-407.

Oberbach A, Bluher M, Wirth H, Till H, Kovacs P, Kullnick Y et al. (2011). Combined proteomic and metabolomic profiling of serum reveals association of the complement system with obesity and identifies novel markers of body fat mass changes. J Proteome Res 10: 4769-4788. 
Pruesse E, Peplies J, Glöckner FO. (2012). SINA: accurate high-throughput multiple sequence alignment of ribosomal RNA genes. Bioinformatics 28: 1823-1829.

Quast C, Pruesse E, Yilmaz P, Gerken J, Schweer T, Yarza P et al. (2013). The SILVA ribosomal RNA gene database project: improved data processing and web-based tools. Nucleic Acids Res 41: D590-D596.

Ramette A. (2007). Multivariate analyses in microbial ecology. FEMS Microbiol Ecol 62: 142-160.

Ravussin Y, Koren O, Spor A, LeDuc C, Gutman R, Stombaugh J et al. (2012). Responses of gut microbiota to diet composition and weight loss in lean and obese mice. Obesity 20: 738-747.

Ridlon JM, Kang D-J, Hylemon PB. (2006). Bile salt biotransformations by human intestinal bacteria. J Lipid Res 47: 241-259.

Robertson BR, O'Rourke JL, Neilan BA, Vandamme P, On SLW, Fox JG et al. (2005). Mucispirillum schaedleri gen. nov., sp. nov., a spiral-shaped bacterium colonizing the mucus layer of the gastrointestinal tract of laboratory rodents. Int J Syst Evol Microbiol 55: 1199-1204.

Schloss PD, Gevers D, Westcott SL. (2011). Reducing the effects of PCR amplification and sequencing artifacts on 16S rRNA-based studies. PLoS One 6: e27310.

Schloss PD, Westcott SL, Ryabin T, Hall JR, Hartmann M, Hollister EB et al. (2009). Introducing mothur: opensource, platform-independent, community-supported software for describing and comparing microbial communities. Appl Environ Microbiol 75: 7537-7541.

Schäfer N, Yu Z, Wagener A, Millrose M, Reissmann M, Bortfeldt $\mathrm{R}$ et al. (2014). Changes in metabolite profiles caused by genetically determined obesity in mice. Metabolomics 10: 461-472.

Serino M, Luche E, Gres S, Baylac A, Bergé M, Cenac C et al. (2012). Metabolic adaptation to a high-fat diet is associated with a change in the gut microbiota. Gut 61: 543-553.

Seyfried F, Li JV, Miras AD, Cluny NL, Lannoo M, Fenske WK et al. (2013). Urinary phenotyping indicates weight loss-independent metabolic effects of Roux-en-Y gastric bypass in mice. J Proteome Res 12: 1245-1253.

Shearer J, Duggan G, Weljie A, Hittel DS, Wasserman DH, Vogel HJ. (2008). Metabolomic profiling of dietary-induced insulin resistance in the high fat-fed C57BL/6J mouse. Diabetes Obes Metab 10: 950-958.

Sonestedt E, Borgquist S, Ericson U, Gullberg B, Olsson H, Adlercreutz $\mathrm{H}$ et al. (2008). Enterolactone is differently associated with estrogen receptor beta-negative and -positive breast cancer in a Swedish nested casecontrol study. Cancer Epidemiol Biomarkers Prev 17: 3241-3251.

Spor A, Koren O, Ley R. (2011). Unravelling the effects of the environment and host genotype on the gut microbiome. Nat Rev Microbiol 9: 279-290.

Steinberg SJ, Dodt G, Raymond GV, Braverman NE, Moser AB, Moser HW. (2006). Peroxisome biogenesis disorders. Biochim Biophys Acta 1763: 1733-1748.

Swann JR, Want EJ, Geier FM, Spagou K, Wilson ID, Sidaway JE et al. (2011). Systemic gut microbial modulation of bile acid metabolism in host tissue compartments. Proc Natl Acad Sci USA 108: 4523-4530.

Tien Poll-The B, Skjeldal O, Stokke O, Poulos A, Demaugre F, Saudubray J-M. (1989). Phytanic acid alpha-oxidation and complementation analysis of classical Refsum and peroxisomal disorders. Hum Genet 81: 175-181.

Timmers R, Rothballer M, Strik DBTB, Engel M, Schulz S, Schloter $M$ et al. (2012). Microbial community structure elucidates performance of Glyceria maxima plant microbial fuel cell. Appl Microbiol Biotechnol 94: 537-548.

Tiribelli C, Ostrow JD. (2005). Intestinal flora and bilirubin. J Hepatol 42: 170-172.

Tominaga S, Nishi K, Nishimoto S, Akiyama K, Yamauchi S, Sugahara T. (2012). (-)-Secoisolariciresinol attenuates high-fat diet-induced obesity in C57BL/6 mice. Food Funct 3: 76-82.

Toye AA, Lippiat JD, Proks P, Shimomura K, Bentley L, Hugill A et al. (2005). A genetic and physiological study of impaired glucose homeostasis control in C57BL/6J mice. Diabetologia 48: 675-686.

Trygg J. (2002). O2-PLS for qualitative and quantitative analysis in multivariate calibration. J Chemometrics 16: 283-293.

Trygg J, Wold S. (2002). Orthogonal projections to latent structures (O-PLS). J Chemometrics 16: 119-128.

Turnbaugh PJ, Hamady M, Yatsunenko T, Cantarel BL, Duncan A, Ley RE et al. (2009). A core gut microbiome in obese and lean twins. Nature 457: 480-484.

Turnbaugh PJ, Ley RE, Mahowald MA, Magrini V, Mardis ER, Gordon JI. (2006). An obesity-associated gut microbiome with increased capacity for energy harvest. Nature 444: 1027-1131.

Tziotis D, Hertkorn N, Schmitt-Kopplin P. (2011). Kendrick-analogous network visualisation of ion cyclotron resonance Fourier transform mass spectra: improved options for the assignment of elemental compositions and the classification of organic molecular complexity. Eur J Mass Spectrom 17: 415-421.

Waldram A, Holmes E, Wang Y, Rantalainen M, Wilson ID, Tuohy KM et al. (2009). Top-down systems biology modeling of host metabotype-microbiome associations in obese rodents. $J$ Proteome Res 8: 2361-2375.

Watanabe M, Horai Y, Houten SM, Morimoto K, Sugizaki T, Arita $\mathrm{E}$ et al. (2011). Lowering bile acid pool size with a synthetic farnesoid $\mathrm{X}$ receptor (FXR) agonist induces obesity and diabetes through reduced energy expenditure. $J$ Biol Chem 286: 26913-26920.

Watanabe M, Houten SM, Mataki C, Christoffolete Ma, Kim BW, Sato $\mathrm{H}$ et al. (2006). Bile acids induce energy expenditure by promoting intracellular thyroid hormone activation. Nature 439: 484-489.

Williams R, Lenz EM, Wilson AJ, Granger J, Wilson ID, Major $\mathrm{H}$ et al. (2006). A multi-analytical platform approach to the metabonomic analysis of plasma from normal and zucker (fa/fa) obese rats. Mol BioSyst 2: 174-183.

Won E-Y, Yoon M-K, Kim S-W, Jung Y, Bae H-W, Lee D et al. (2013). Gender-specific metabolomic profiling of obesity in leptin-deficient ob/ob mice by $1 \mathrm{H}$ NMR spectroscopy. PLoS One 8: e75998.

Woting A, Clavel T, Loh G, Blaut M. (2010). Bacterial transformation of dietary lignans in gnotobiotic rats. FEMS Microbiol Ecol 72: 507-514.

Xie B, Waters MJ, Schirra HJ. (2012). Investigating potential mechanisms of obesity by metabolomics. J Biomed Biotechnol 2012: 805683. 
Xie Z, Li H, Wang K, Lin J, Wang Q, Zhao G et al. (2010). Analysis of transcriptome and metabolome profiles alterations in fatty liver induced by high-fat diet in rat. Metabolism 59: 554-560.

Xu J, Liu C, Cai S, Dong J, Li X, Feng J et al. (2013). Metabolomic profilings of urine and serum from high fat-fed rats via $1 \mathrm{H}$ NMR spectroscopy and pattern recognition. Appl Biochem Biotechnol 169: 1250-1261.
Zhang C, Zhang M, Wang S, Han R, Cao Y, Hua W et al. (2010). Interactions between gut microbiota, host genetics and diet relevant to development of metabolic syndromes in mice. ISME J 4: 232-241.

Zhao LC, Zhang XD, Liao SX, Gao HC, Wang HY, Lin DH. (2010). A metabonomic comparison of urinary changes in Zucker and GK rats. I Biomed Biotechnol 2010: 431894.

Supplementary Information accompanies this paper on The ISME Journal website (http://www.nature.com/ismej) 